\title{
Laboratory diagnostics and pathogenesis of rheumatoid arthritis - the past and the present
}

\author{
Corresponding author: \\ Magdalena Kuligowska-Prusińska, PhD \\ Department of Laboratory Medicine, \\ Nicolaus Copernicus University, \\ Collegium Medicum in Bydgoszcz \\ Sklodowskiej-Curie 9 St. \\ 85-094 Bydgoszcz, Poland \\ E-mail: magdalenakuligowska@wp.p
}

Medical Research Journal 2017; Volume 2, Number 4, 128-134 10.5603/MRJ.2017.0017 Copyright (C) 2017 Via Medica ISSN 2451-2591

\begin{abstract}
Rheumatoid arthritis (RA) is one of the most common systemic connective tissue diseases of autoimmune origin and is characterised by chronic inflammation of joints. The aetiology of this disease has not been fully understood yet. A crucial role in the development of RA is played by multiple genetic (shared epitope, HLA-DR genes, family predisposition) and environmental factors (smoking, female-specific factors, bacteria, viruses and mucosal inflammation, periodontal and lung diseases). The purpose of this article is to review the latest data on the pathogenesis of this disease and biomarkers used in the diagnostics of RA. RA is associated with the production of autoantibodies, among which rheumatoid factor (RF) and anti-citrullinated protein (anti-CCP) antibodies are included in the new classification criteria of RA. So far, anti-CCP is the best marker of RA; its high sensitivity and specificity have been helpful in diagnostics and monitoring disease activity and the development of more aggressive disease, as well as the pharmacological treatment used. Recently, anti-carbamylated (anti-carP) antibodies and calprotectin have been described in RA, which also appears to be promising in the diagnostics of this disease.
\end{abstract}

Keywords: rheumatoid arthritis, rheumatoid factor, antibodies, calprotectin, classification criteria

Med Res J 2017; 2 (4): 128-134

\section{Introduction}

Rheumatoid arthritis (RA) is a chronic immunological-dependent inflammatory disease of the joints in which cartilage and bone damage as well as extra-articular manifestations and organ changes, such as pulmonary involvement and cardiovascular complications, occur [1]. RA is one of the most common systemic connective tissue diseases of autoimmune origin. Although it is present in $0.5-2 \%$ of the general population, it is characterised by quite high morbidity and mortality; in the United States alone, there are 1.3 million people suffering from RA, and the costs associated with the disease amount to 19.3 billion dollars [2-3]. RA can be diagnosed at any age; however, the highest incidence is seen between $40-50$ years of age, and the number of patients increases with age.

In this paper, we review the latest data on laboratory markers and pathogenesis of rheumatoid arthritis with particular emphasis on antibodies, their diagnostic and prognostic value, and many factors involved in the development of RA.

\section{Pathogenesis and risk factors for RA}

The aetiopathogenesis of the disease has not yet been recognised, but an important role in the development of RA is attributed to the immunological mechanisms, especially in patients with genetic predispositions, and the involvement of environmental factors. It has been proven that a positive family history has been associated with an increased risk of RA $[1,4]$. Due to the three-fold more frequent incidence of the disease in women, the participation of female-specific factors in the pathogenesis of RA is also taken into account. Among the factors that increase the risk of RA are early menopause or polycystic ovary syndrome, whereas hormone replacement therapy and oral contraception are protective factors. The exact mechanism is unknown, but an early preclinical effect of hormones in the development of RA is probable. It is thought that exogenous hormones lead to a decrease in the secretion of endogenous hormones, and thus a decrease in the risk of developing the disease [4]. Furthermore, the contribution of various microbes, including bacteria or 
bacterial antigens, viruses, as well as mucosal inflammation, to the development of RA has been observed [5]. Moreover, recent years have also demonstrated a relationship between the occurrence of RA and periodontal disease, and the Porphyromonas gingivalis (P.ging) pathogen is responsible for the production of bacterial PAD enzymes that mediate citrullination reactions of RA-related antibodies [1,5]. The risk of developing the disease is also influenced by lifestyle, including smoking and poor diet leading to obesity. Many studies have shown a strong association between cigarette smoke and RA risk, increased protein citrullination, and therefore anti-CCP positive RA, but also the presence of RF before disease symptoms occur [6-7]. This factor is a potential cause of increased antibody synthesis, especially in patients with a genetic predisposition to RA. In addition, tobacco smoke affects the growth of periodontal and lung diseases, which can lead to inflammatory and autoimmune changes initiating the systemic action that is the development of RA [4,7]. Various dietary factors, including high intake of sugar, cheese, red meat, or protein increase the risk of developing RA, while a healthy diet based on low consumption of sugar and animal fats, and rich in fruits and vegetables, reduces the risk of disease [4].

Genetic factors affect the development of RA in less than $50 \%$ of cases [5]. Particularly susceptible to RA are those patients with a shared epitope (SE) of the HLA-DR class II, the major histocompatibility complex $(\mathrm{MHC})$. It is suggested that alleles with the same sequence of shared epitope HLA-DRB1 are a genetic factor predisposing to the disease [1,7]. In addition, some HLA-DR genes are associated with a more aggressive form of the disease and increased mortality of patients [1]. Many specific genetic loci are also responsible for the increased risk of RA, e.g. protein tyrosine phosphatase gene (PTPN22) or signal transducer and activator of transcription 4 (STAT4) [4-5]. The relationship between the presence of anti-CCP antibodies and a shared epitope in RA patients is also described $[4,8]$. It emerges that in patients with shared epitope, the concentration of anti-CCP significantly depends on environmental factors, i.e. smoking. The occurrence of HLA-DR4 is associated with the presentation of the antigen and the initiation of an autoimmune response, leading to arthritis and, in particular, to synovitis. Active T lymphocytes can stimulate other cells to produce many inflammatory mediators, e.g. cytokines, enzymes, and antibodies. An important role in this process is played by pro-inflammatory cytokines, i.e. IL-1, IL-6, and TNF- $\alpha$, which, due to the connection with RA, are not only the objective of research, but also of new biological treatment [4].

\section{Serological diagnostics}

The search for specific immune mechanisms involved in the development of the disease has resulted in the detection of antibodies in RA patients. The prevalence of autoantibodies is found in the majority of RA patients [9]. Therefore, the basis for the immunodiagnostics of rheumatic diseases are currently serological tests in which antibodies are detected. To date, relatively few antibodies have been well-established in the diagnostics of autoimmune rheumatic diseases. This is most frequently due to the fact that these markers are not always sufficiently sensitive and specific indicators of a clinically defined disease. To date, only two antibodies have been included in the classification criteria for RA $[2,10]$.

\section{Rheumatoid factor}

Rheumatoid factor (RF) is known to play an important role in the pathogenesis of RA. RF is an autoantibody directed against Fc fragments of the human immunoglobulin class $\mathrm{G}$ ( $\mathrm{IgG}$ ) and classical RF, i.e. RF IgM was detected in 1949 by Eric Waaler and Harry Rose. RF can also be found in other classes of immunoglobulins, but their determination has only an auxiliary value $[8,11]$.

The rheumatoid factor remains the main serological marker of RA, on the basis of which the seropositive (the presence RF IgM) or seronegative (no RF IgM) subtypes of the disease are distinguished. In addition to the stimulation of the immune system and the formation of immune complexes, these factors also participate in the complement activation. RF combined with immune complexes intensifies inflammatory processes, leading to the destruction of cartilage and the joint as well as to the development of extra-articular RA. Increased secretion of RFs by various cells, primarily in the bone marrow and synovial fluid, correlates with the severity of lesions [11].

The highest level of RF IgM is detected in RA patients; therefore, the determination of RF in RA has a certain prognostic value [9]. Seropositive RA is usually characterised by a more severe course of the disease, faster destruction of joint tissues, and a greater inclination to extra-articular lesions compared to seronegative RA. In particular, the presence of RF IgG or RF IgA is associated with the occurrence of extra-articular manifestation [12]. It has been demonstrated that the level of RF also varies depending on the activity of the disease process, which is used in monitoring the course of the disease and the effectiveness of the treatment applied. In addition, it can also appear in the serum of patients before the onset of symptoms. Furthermore, it has been observed that patients with high RF IgA or RF IgG titres are also at increased risk of RA [3-4]. 
It should also be remembered that RF may occur in other connective tissue diseases, which significantly reduces its diagnostic value as a specific marker. $R F$ is also present in $3-5 \%$ of healthy individuals, especially after 60 years of age $[3,8]$.

\section{Anti-citrullinated protein antibodies (ACPAs)}

For some time, in the laboratory diagnostics of RA the determination of antibodies against cyclic citrullinated peptide (anti-CCP), which were described for the first time in 1998 by Schellekens et al., has been used [3].

Their studies demonstrated that sera of RA patients bind antigenic determinants containing citrulline, which is the result of the post-translational modification of arginine by peptidylarginine deiminase (PAD) enzyme in citrullination reaction $[3,8,13]$.

The oxygen group plays a key role in the process of recognising modified peptides by the human immune system as alien, which in turn leads to the synthesis of ACPA. Therefore, this group also includes antibodies against modified citrullinated vimentin (anti-MCV), anti-filaggrin antibodies (AFA), antibodies against the perinuclear factor (APF), anti-keratin antibodies (AKA), and anti-Sa antibodies (anti-Sa) [8]. What seems helpful is to determine anti-MCV antibodies that identify patients with anti-CCP2 (-) and RF (-), which is important in clinical practice. The presence of anti-MCV antibodies is detected in $10 \%$ of patients with CCP (-) and $30 \% \operatorname{RF~IgM~(-)~}[8,14]$.

So far, five isoforms of the human PAD enzyme have been identified (PAD1, PAD2, PAD3, PAD4-5, and PAD6) [8]. It has been shown that the PAD4 gene variant, which codes one of the PAD enzyme isotypes by increasing the enzymatic activity, increases the susceptibility to RA $[8,13]$. Furthermore, the expression of the PAD enzyme and the protein citrullination are associated with the synovium. It has been demonstrated that almost half of patients with RA have anti-PAD4, which is associated with higher levels of anti-CCP, RF IgM, and IgG as well as higher erythrocyte sedimentation rate (ESR) and more advanced lesions in joints. It is therefore suggested that PAD4 may act as an autoantigen that plays a role in the pathogenesis of RA [8].

Anti-CCP antibodies most often belong to the $\lg G$ class and are produced locally in the RA-affected synovium [8]. Probably, inflammatory processes, intensified apoptosis, or necrosis result in local lesions, as a result of which a modification emerges that is associated with the formation of antigenic epitopes, e.g. citrullination. Autoantigens modified in this way may stimulate the production of antibodies, which causes an autoimmune reaction.

The development of an enzymatic assay (ELISA) and the use of cyclic (instead of linear) peptide containing ci- trulline in the first-generation tests (CCP I) increased the sensitivity of the method from $49 \%$ to $68 \%$. In contrast, the use of highly purified synthetic peptides containing citrulline in the second-generation tests (CCP II) or immunobiochemical analysers resulted in a sensitivity of $75-82 \%$ and specificity of $98 \%$ [3].

Considering their high sensitivity and specificity, anti-CCP is essentially the best marker of RA and is not practically detected in healthy people (0-1\%); only a few per cent are found in other rheumatic and infectious diseases (1-8\%). However, they are detected in $40-60 \%$ of patients with early-stage disease, which is important in the diagnosis of early RA. Many studies indicate a high predictive value for the identification of patients in pre-clinical RA, who will develop a disease in the future, because the appearance of anti-CCP may precede the first clinical symptoms. Studies show that the risk of developing RA is higher in individuals in whom anti-CCP was detected [9]. In addition, the low level of anti-CCP is a predictor of a good response to the treatment in patients with early RA [15]. Moreover, their determination is extremely helpful in the diagnostics of seronegative RA because they are detected in ca. $35-40 \%$ of patients in whom no RF is found. The prognostic value of anti-CCP is also emphasised, the presence and concentration of which correlate with the occurrence of RF IgM and disease activity, as well as the severity of joint destruction and the development of more aggressive disease [2]. In patients with anti-CCP $(+)$, the occurrence of joint erosion is more frequent in comparison with patients with anti-CCP (-). Moreover, high concentration of anti-CCP correlates with the highest radiological progression [16]. It is also interesting that the anti-CCP concentration strongly correlates with smoking, and the highest values of anti-CCP have been demonstrated in active smokers with RA. In contrast to the level of anti-CCP, the concentration of RF was not different in smokers and non-smokers with RA [8].

To increase the diagnostic sensitivity and specificity, it is recommended to determine several markers in rheumatoid arthritis (Tab. 1). It has been proven that the combined determination of several indicators in RA patients is characterised by higher sensitivity and specificity than the analysis of individual markers, which turns out to be more useful in laboratory diagnostics of this disease. Many studies indicate anti-CCP and rheumatoid factor as the most important predictors of RA [1-3]. The introduction of routine anti-CCP antibody determination and their high clinical utility have resulted in anti-CCP antibodies being one of the most useful parameters in the diagnosis of RA, leading to the need to extend the diagnostic criteria of RA [8]. 
Table 1. A comparison of the sensitivity and specificity of antibodies in RA [8].

\begin{tabular}{lcc}
\hline Antibodies & Sensitivity [\%] & Specificity [\%] \\
\hline anti-CCP1 & 72 & 80 \\
anti-CCP2 & 82 & 98 \\
anti-CCP3 & 82 & 92 \\
anti-MCV & 40 & 98 \\
RF IgM & $66-80$ & 84 \\
APF & $50-70$ & 90 \\
anti-AKA & 45 & 94 \\
anti-AFA & 60 & 98 \\
anti-Sa & $22-40$ & 99 \\
RA33 & 36 & 87 \\
anti-carP & 29 & $95-99$ \\
\hline
\end{tabular}

Table 2. Current classification criteria of RA according to ACR / EULAR of 2010 [10,17].

\begin{tabular}{lc}
\hline A. Joint involvement & Points \\
\hline of 1 large joint & 0 \\
of $2-10$ large joints & 1 \\
of $1-3$ small joints & 2 \\
of $4-10$ small joints & 3 \\
of $>10$ joints (at least 1 small joint) & 5 \\
\hline
\end{tabular}

\section{B. Serological parameters}

RF (-) and anti-CCP (-) 0

Low-positive RF and/or anti-CCP 2

High-positive RF and/or anti-CCP

2

3

\section{Acute phase reactants}

$\mathrm{CRP}$ or $\mathrm{OB}$ within the reference range

0

$\mathrm{CRP}$ or $\mathrm{OB}>$ the reference range

\section{Duration of arthritis}

$<6$ weeks

$>6$ weeks

1

Interpretation: The patient can be assigned a maximum of 10 points. It was established that the presence of at least six points allows diagnosis of established RA. The more points obtained, the more likely it is that arthritis will be chronic and erosive. With a time shorter than six weeks, a large number of affected joints is not enough; it is necessary to find an increased acute phase indicator or a positive result of at least one serological parameter. In the case of persistent inflammation $>6$ weeks, the diagnosis can be established in patients in whom $\mathrm{RF}$ and/or anti-CCP are not detected, and acute phase indicators are normal.

\section{Classification criteria of RA}

Criteria for the diagnosis of RA developed by the American College of Rheumatology (ACR) have been evolving since the 1960s, when the first criteria consisted of 11 symptoms and included tests invasive for the patient, i.e. histopathological assessment of the synovium and rheumatoid nodule or analysis of the synovial fluid. Thus, on their basis, the diagnosis could only be probable, certain, or classical depending on the occurring symptoms. Subsequent criteria included seven symptoms, among them as many as five clinical, one radiological, and one immunological - $\mathrm{RF} \operatorname{lgM}$. To diagnose the patient, at least four criteria had to be met. Diagnostic difficulties were usually because these criteria fulfilled their role only in patients with an already established disease, and did not allow the diagnosis of early or atypical cases. Therefore, the European League Against Rheumatism (EULAR) developed new classification criteria for RA, which include four groups of parameters, i.e. the type and number of joints affected, serological parameters (anti-CCP, RF), determination of acute phase indicators (CRP, ESR), and duration of arthritis (Tab. 2) $[1,8,10,12]$.

Currently, according to these classification criteria, anti-CCP are the gold standard in the diagnostics of RA. The basic laboratory parameters, i.e. the erythrocyte sedimentation rate (ESR) and C-reactive protein (CRP), have also been included in these criteria, despite the fact that these are non-specific indicators of RA. The values of ESR and CRP are increasing in patients with $\mathrm{RA}$, which correlates with the clinical condition, and are used to monitor the severity of the inflammatory process and the effectiveness of the treatment applied. Increasing the CRP level may also indicate an exacerbation or recurrence of the disease after the remission period, so its determination is useful for assessing the disease activity score (DAS) (Tab. 3) $[3,10,11,15,17]$.

Due to the chronic nature of the disease and aggressive treatment it is also necessary to monitor the general condition of the RA patient; therefore, it is indispensable to perform basic laboratory tests, i.e. blood counts and urine tests.

It is also important to assess the parameters of kidney and liver functions, taking into account the hepatotoxic and neurotoxic effects of many anti-rheumatic drugs.

Table 3. The importance of introducing the current RA classification criteria [3].

Early diagnosis of RA

Assessment of potential disease progression risk and predisposition to erosive RA

Diagnostics of patients with negative RF and other inflammatory diseases of joints

Early treatment - higher probability of remission of RA 


\section{New laboratory biomarkers in RA}

Apart from antibodies against citrullinated proteins (ACPA), additional markers are still being sought that would help in the diagnostics of RA, especially in seronegative patients. The focus of researchers is primarily biomarkers useful for assessing disease activity and prognosis of joint damage in patients with RA. Recently, anti-carbamylated (anti-carP) antibodies and calprotectin have been described in RA, which also appears to be promising in the diagnosis of this disease [18-30].

\section{Antibodies against carbamylated proteins (anti-carP)}

Anti-carbamylated antibodies (anti-carP) are directed against post-translationally modified proteins that were found in approximately $45 \%$ of RA patients and, importantly, in $30 \%$ of ACPA-negative patients [18]. Both carbamylation and citrullination are post-translational modifications that result in the emergence of carbamylated or citrullinated proteins in which positively charged amino acids are replaced by neutral amino acids. Carbamylation, in contrast to citrullination, which occurs under the influence of the PAD enzyme, is a non-enzymatic chemical modification in which cyanate binds to molecules containing basic amino or thiol groups. Cyanate occurs naturally in the body and is in equilibrium with urea. Under physiological conditions, it can be generated from urea only at low concentration or come from the environment, e.g. from exhaust fumes [20]. Therefore, in the state of equilibrium, due to the low concentration of cyanate, intense carbamylation of proteins does not occur. On the other hand, an increased carbamylation reaction was first observed in patients with elevated levels of urea, i.e. patients with renal diseases, and then in cardiovascular diseases. The carbamylation reaction has recently been described in immune tolerance disorders, and therefore anti-carP antibodies have been identified in rheumatoid arthritis. Interestingly, cigarette smoke increases the concentration of cyanate, which may contribute to the increase in carbamylation of proteins [19]. However, no association was observed between the presence of anti-carP antibodies and the presence of PTPN22 gene polymorphism or HLA-DRB1-SE alleles of MHC, which may indicate another biological mechanism resulting in the production of anti-carP antibodies compared to anti-CCP antibodies. However, the relationship between HLA-DRB1 * 03 and positive test for anti-carP in RA patients is considered because, in the anti-carP $(+)$ group, a stronger correlation was observed than in anti-carP (-) patients. In relation to genetic and environmental risk factors, the participation of yet other genes cannot be potentially excluded, which may be important in the development of RA in anti-carP (+) patients [21].

It is believed, however, that increased carbamylation occurs in inflammation due to myeloperoxidase (MPO) released by active neutrophils. It has been shown that MPO has the ability to convert thiocyanate to cyanate, thereby allowing enhanced carbamylation. Hence, it is presumed that in RA carbamylation may be a naturally occurring reaction to synovitis $[20,23]$.

It has been demonstrated that lysine residues, and not arginine as in the citrullination reaction, are subject to carbamylation, whereas the obtained amino acid is homocitrulline, which is very similar to citrulline $[9,22-23]$.

Currently, the direct participation of anti-carP in the pathogenesis of RA is unknown. Antibodies recognising homocitrulline were found in anti-CCP $(+)$ and anti-CCP $(-)$ patients in both IgG and IgA classes in two independent studies [23].

It is important that in ACPA-negative patients, the occurrence of anti-carP antibodies was associated with more advanced progression of lesions visible in radiological examinations. In addition, anti-carP may appear many years before RA is diagnosed, and disease progression in patients with joint pain, regardless of anti-CCP and RF IgM, can be predicted on the basis of detecting the antibodies. It has been demonstrated that the presence of anti-carP in patients with joint pain is associated with an increased risk of developing RA. In another study, these antibodies were detected in 44 to $67 \%$ of RA ACPA (-) patients. Moreover, it has been demonstrated that anti-carP may be a predictor of joint damage, as well as of the future development of RA, not only in patients with joint pain, but also in potentially healthy blood donors. Although the sensitivity of these antibodies is quite low, they have very high specificity. Therefore, their determination seems to be important in the diagnostics of undifferentiated inflammation of the joints and in seronegative patients. Moreover, simultaneous assessment of anti-carP and ACPA antibodies may be helpful in identifying patients at risk and those with early-stage disease [19].

Anti-carP antibodies can occur in both adult and paediatric patients, which is why they were found in juvenile idiopathic arthritis (JIA), which is one of the most common childhood rheumatic diseases [23] .

The so far promising research results indicate that anti-carP antibodies may prove to be a potentially useful biomarker in the diagnostics of RA, in particular in the risk assessment of erosive lesions and progression of lesions visible in radiological examination in RA patients with RA [19-23].

\section{Calprotectin}

Calprotectin, also known also as myeloid-related protein MRP8/14, protein S100A8/A9, the L1 protein, or calgranulin $A$ and $B$, belongs to the $S 100$ protein family and 
consists of two calcium-binding subunits that form the heterodimer complex S100A8/S100A9 (MRP8/MRP14) [26,28-29]. To date, over 20 proteins belonging to the S100 family have been identified [29]. The MRP8/14 molecular weight is determined at $36.5 \mathrm{kDa}$ and the biological half-life in plasma is five hours. Although calprotectin was isolated in 1980 by Fagerhola et al., the term was later proposed to reflect the role of this protein in epithelial protection as well as its fungicidal and bactericidal activity [28]. Unlike other $\mathrm{S} 100$ family proteins, besides $\mathrm{Ca}^{2+}$ ions, calprotectin also binds $\mathrm{Zn}^{2+}$ ions, which affects the process of apoptosis. The formation of calprotectin subunits occurs in cells of the granulocytic and monocytico-phagocytic systems. However, after stimulation with many factors, e.g. proinflammatory cytokines, lipopolysaccharides, or components of the complement system, protein molecules are released outside the cell.

Calprotectin is one of the most significant proteins involved in the regulation of the immune system and the inflammatory process. It is considered to be an important proinflammatory factor of the unspecific immune response that acts through Toll-like receptor-4. Calprotectin is classified as an endogenous molecule released mostly by immune cells, and tissue and cell damage. It is known that this protein is secreted in non-classical pathway and is not synthesised de novo [30]. Calprotectin secreted from activated granulocytes and macrophages shows pro-inflammatory activity in vitro against phagocytes and endothelial cells, and in vivo causes inflammation [28].

The increase in calprotectin concentration is found in various body fluids, i.e. serum, faeces, urine, saliva, sputum, synovial fluid, and amniotic fluid. So far, it has been demonstrated that increased levels of this protein occur in chronic intestinal inflammation, cystic fibrosis, psoriasis, and multiple sclerosis as well as rheumatic diseases, i.e. rheumatoid arthritis, systemic lupus erythematosus, or Sjögren's syndrome. In addition, due to the low molecular weight, this protein can diffuse from the arthritis into the bloodstream, which makes it possible to determine the level of calprotectin in serum or plasma. A good correlation was also found between the concentration of calprotectin in the plasma and synovial fluid [24]. Higher concentrations of calprotectin have been detected in patients with rheumatoid arthritis compared to osteoarthritis or spondyloarthritis [24,28-29]. Moreover, elevated levels of calprotectin have been shown in RF-positive patients than in RF-negative RA patients [29]. Furthermore, the relationship between the level and the presence of anti-CCP and RF antibodies has been described [24]. Additionally, ACPA, RF IgM, or IgA-positive patients had higher levels of calprotectin than patients with negative RA antibodies [29]. Regarding this, it was demonstrated that the level of calpro- tectin correlates with disease activity and inflammation of joints in patients with RA. The relationship between calprotectin and clinical and laboratory indicators of arthritis, such as Disease Activity Score, C-reactive protein, erythrocyte sedimentation rate, or rheumatoid factor, has been demonstrated [24,26-27,29].

Furthermore, high concentration of this protein is a prognostic factor for the radiological progression of the disease independent of CRP and OB [24-25,27]. This protein is associated with joint damage and is considered a better indicator of ultrasound synovitis in RA patients than routine laboratory parameters, i.e. OB or CRP [26-27]. In experimental models, calprotectin has been shown to contribute to the degradation of cartilage and bone by stimulating the secretion of enzymes degrading the matrix and activation of osteoclast formation [30]. Recently, it has been emphasised that calprotectin can be a potentially more sensitive biomarker of rheumatoid disease activity than traditional indicators of inflammation, i.e. CRP, because it directly determines the inflammation in the synovium [28].

It is suggested that the determination of calprotectin level could be used also to monitor the therapeutic effect in patients with RA $[28,30]$. It has been demonstrated that conventional treatment with disease-modifying drugs (DMARDs) as well as a biological therapy significantly decreases the concentration of calprotectin in RA patients [30].

Moreover, numerous studies have shown a relationship between the level of calprotectin and disease activity in patients with other rheumatic diseases, i.e. Still's disease, psoriatic arthritis, primary Sjögren's syndrome, or juvenile idiopathic, which makes it possible to use it in the diagnosis of other rheumatic diseases as well [28].

\section{Conclusions}

Advances in the field of rheumatology, immunology, and laboratory diagnostics in recent decades have contributed to a better understanding of the mechanisms responsible for the development and pathogenesis of rheumatoid arthritis, and thus a better diagnosis and treatment of patients with RA. It is interesting that many genetic and environmental factors, i.e. smoking, may influence the risk of developing rheumatoid arthritis in the future, as well as the emergence of antibodies characteristic for the disease, such as anti-CCP. Replacing the 1987 criteria with the new classification criteria of RA in 2010 gave a greater opportunity to recognise early cases of the disease - especially in seronegative patients, and to introduce treatment at an earlier stage of the disease, and thus it increased the chances of remission of the disease. Due to the progressive and 
insidious nature of RA, it is important to constantly monitor the course of the disease and the pharmacological treatment used. Although anti-CCP antibodies are the gold standard in the diagnostics of RA, it is necessary to search for new biomarkers for assessing disease activity and prognosis of joint damage in patients with RA. Anti-carP antibodies and calprotectin also appear promising in the diagnosis of RA, assessment of disease severity and joint damage, as well as further progression and potential therapeutic response, but their exact involvement in RA still requires extensive research.

\section{References}

1. Smolen JS, Aletaha D, Mclnnes IB. Rheumatoid arthritis. Lancet. 2016; 388(10055): 2023-2038, doi: 10.1016/S0140-6736(16)30173-8, indexed in Pubmed: 27156434.

2. Shafrin J, Tebeka MG, Price K, et al. The Economic Burden of ACPA-Positive Status Among Patients with Rheumatoid Arthritis. J Manag Care Spec Pharm. 2018; 24(1): 4-11, doi: 10.18553/jmcp.2017.17129, indexed in Pubmed: 29290168.

3. Taylor P, Gartemann J, Hsieh J, et al. A systematic review of serum biomarkers anti-cyclic citrullinated Peptide and rheumatoid factor as tests for rheumatoid arthritis. Autoimmune Dis. 2011; 2011: 815038, doi: 10.4061/2011/815038, indexed in Pubmed: 21915375.

4. Deane K, Demoruelle M, Kelmenson L, et al. Genetic and environmental risk factors for rheumatoid arthritis. Best Practice \& Research Clinical Rheumatology. 2017; 31(1): 3-18, doi: 10.1016/j.berh.2017.08.003.

5. Roszyk E, Puszczewicz M. Role of human microbiome and selected bacterial infections in the pathogenesis of rheumatoid arthritis. Reumatologia. 2017; 55(5): 242-250, doi: 10.5114/reum.2017.71641 indexed in Pubmed: 29332963.

6. Hedström AK, Stawiarz L, Klareskog L, et al. Smoking and susceptibility to rheumatoid arthritis in a Swedish population-based case-control study. Eur J Epidemiol. 2018 [Epub ahead of print], doi: 10.1007/s10654018-0360-5, indexed in Pubmed: 29387991.

7. Joshua V, Chatzidionisyou K, Catrina Al. Role of the lung in individuals at risk of rheumatoid arthritis. Best Pract Res Clin Rheumatol. 2017 31(1): 31-41, doi: 10.1016/j.berh.2017.08.002, indexed in Pubmed 29221596

8. Farid SSh, Azizi G, Mirshafiey A. Anti-citrullinated protein antibodies and their clinical utility in rheumatoid arthritis. Int J Rheum Dis. 2013 16(4): 379-386, doi: 10.1111/1756-185X.12129, indexed in Pubmed 23992255

9. Falkenburg WJJ, van Schaardenburg D. Evolution of autoantibody responses in individuals at risk of rheumatoid arthritis. Best Pract Res Clin Rheumatol. 2017; 31(1): 42-52, doi: 10.1016/j.berh.2017.07.005 indexed in Pubmed: 29221597.

10. Aletaha D, Neogi T, Silman AJ, et al. 2010 Rheumatoid arthritis classification criteria. Arthritis Rheum. 2010; 62: 2569-2581.

11. Matuszewska A, Madej M, Wiland P. Markery immunologiczne reumatoidalnego zapalenia stawów. Postepy Hig Med Dosw (online. 2016; 70: 251-257

12. Gavrilă BI, Ciofu C, Stoica V. Biomarkers in Rheumatoid Arthritis, what is new? J Med Life 2016: 9(2): 144-148, indexed in Pubmed: 27453744

13. Schett $\mathrm{G}$. The role of ACPAs in at-risk individuals: Early targeting of the bone and joints. Best Pract Res Clin Rheumatol. 2017; 31(1): 53-58 doi: 10.1016/j.berh.2017.09.015, indexed in Pubmed: 29221598

14. van der Linden MPM, van der Woude D, loan-Facsinay A, et al. Value of anti-modified citrullinated vimentin and third-generation anti-cyclic citrullinated peptide compared with second-generation anti-cyclic citrullinated peptide and rheumatoid factor in predicting disease outcome in undifferentiated arthritis and rheumatoid arthritis. Arthritis Rheum. 2009; 60(8): 2232-2241, doi: 10.1002/art.24716, indexed in Pubmed: 19644872

15. Brzustewicz E, Henc I, Daca A, et al. Autoantibodies, C-reactive protein, erythrocyte sedimentation rate and serum cytokine profiling in monitoring of early treatment. Cent Eur J Immunol. 2017; 42(3): 259-268, doi: 10.5114/ceji.2017.70968, indexed in Pubmed: 29204090.

16. van Oosterhout M, Bajema I, Levarht EWN et al. Differences in synovial tissue infiltrates between anti-cyclic citrullinated peptide-positive rheumatoid arthritis and anti-cyclic citrullinated peptide-negative rheumatoid arthritis. Arthritis Rheum. 2008; 58(1): 53-60, doi: 10.1002/art.23148, indexed in Pubmed: 18163491.

17. Šenolt L, Grassi W, Szodoray P. Laboratory biomarkers or imaging in the diagnostics of rheumatoid arthritis? BMC Med. 2014; 12: 49, doi: 10.1186/1741-7015-12-49, indexed in Pubmed: 24642071

18. Shi J, van de Stadt LA, Levarht EW, et al. Anti-carbamylated protein antibodies are present in arthralgia patients and predict the development of rheumatoid arthritis. Arthritis Rheum. 2013; 65(4): 911-915, doi: 10.1002/art.37830, indexed in Pubmed: 23279976.

19. Jiang $X$, Trouw LA, van Wesemael TJ, et al. Anti-CarP antibodies in two large cohorts of patients with rheumatoid arthritis and their relationship to genetic risk factors, cigarette smoking and other autoantibodies. Ann Rheum Dis. 2014; 73(10): 1761-1768, doi: 10.1136/annrheumdis-2013-205109, indexed in Pubmed: 24812286.

20. Yee A, Webb T, Seaman A, et al. Anti-CarP antibodies as promising marker to measure joint damage and disease activity in patients with rheumatoid arthritis. Immunol Res. 2015; 61(1-2): 24-30, doi: 10.1007/s12026-014-8560-x, indexed in Pubmed: 25391608

21. Bax M, Huizinga TWJ, Toes REM. The pathogenic potential of autoreactive antibodies in rheumatoid arthritis. Semin Immunopathol. 2014; 36(3): 313-325, doi: 10.1007/s00281-014-0429-5, indexed in Pubmed: 24763532

22. Shi J, van Veelen PA, Mahler M, et al. Carbamylation and antibodies against carbamylated proteins in autoimmunity and other pathologies. Autoimmun Rev. 2014; 13(3): 225-230, doi: 10.1016/j. autrev.2013.10.008, indexed in Pubmed: 24176675.

23. Kumar S, Pangtey G, Gupta R, et al. Assessment of anti-CarP antibodies, disease activity and quality of life in rheumatoid arthritis patients on conventional and biological disease-modifying antirheumatic drugs. Reumatologia. 2017; 55(1): 4-9, doi: 10.5114/reum.2017.66680, indexed in Pubmed: 28386136

24. Jonsson MK, Sundlisæter NP, Nordal HH, et al. Calprotectin as a marker of inflammation in patients with early rheumatoid arthritis. Ann Rheum Dis. 2017: 76(12): 2031-2037, doi: 10.1136/annrheumdis-2017-211695, indexed in Pubmed: 28814431.

25. Elnady B, Soliman A, Shaker RM, et al. Potential role of calprotectin as a monitoring biomarker for clinical and sonographic activity and treatment outcome in recent-onset rheumatoid arthritis. Egyptian Rheumatology and Rehabilitation. 2016; 43(3): 143, doi: 10.4103/1110-161x.189824.

26. Mansour HE Abdullrhman MA, Mobasher SA et al Serum Calprotectin in Rheumatoid Arthritis: A Promising Diagnostic Marker, How Far Is It Related to Activity and Sonographic Findings? Journal of Medical Ultrasound. 2017; 25(1): 40-46, doi: 10.1016/i.jmu.2016.11.001.

27. Hurnakova J, Hulejova H, Zavada J, et al. Serum Calprotectin Discriminates Subclinical Disease Activity from Ultrasound-Defined Remission in Patients with Rheumatoid Arthritis in Clinical Remission. PLoS One. 2016; 11(11): e0165498, doi: 10.1371/journal.pone.0165498, indexed in Pubmed: 27832086

28. Kopeć-Mędrek M, Widuchowska M, Kucharz EJ. Calprotectin in rheumatic diseases: a review. Reumatologia. 2016; 54(6): 306-309, doi: 10.5114/reum.2016.64907, indexed in Pubmed: 28115781

29. Kang KY, Woo JW, Park SH. S100A8/A9 as a biomarker for synovial inflammation and joint damage in patients with rheumatoid arthritis. Korean J Intern Med. 2014; 29(1): 12-19, doi: 10.3904/kjim.2014.29.1.12, indexed in Pubmed: 24574827

30. Šenolt L Calprotectin (a S100 protein) as a sensitive biomarker for rheumatoid arthritis: new perspectives for an old finding. International Journal of Clinical Rheumatology. 2012; 7(2): 127-129, doi: 10.2217/ijr.12.1. 